\title{
Editorial
}

International Archives of
Allergy
Immunology

\section{The Use of lonizers to Destroy Allergens: Past, Present and Future Research}

\author{
Neil Goodman
}

Fluent Science, Brussels, Belgium

Although previous research has shown that the allergens Der p 1, Der $\mathrm{f} 1$ and Fel $\mathrm{d} 1$ are destroyed after exposure to corona discharge, it remains to be seen how this occurred [1,2]. It is essential that we know how these allergens are being inactivated so that we can be sure that they are made safe and to possibly improve the efficacy of the treatment. However, at present, there is a dearth of information on the chemical reactions that occur when proteins encounter the products of corona discharge.

In this issue of International Archives of Allergy and Immunology, Kawamoto et al. [3] report on the observed decrease in allergenicity of allergens when treated with positive and negative ions. In this instance, the allergens under study were from Japanese cedar (Cryptomeria japonica) pollen (JCP): Cry $\mathrm{j} 1$ and Cry j 2. In their bench testing, atomized JCP extract was treated with ion-generating devices consisting of a ceramic dielectric plate, a high-voltage applied electrode and an earth electrode in an enclosed experimental system that subjected the atomized JCP solution to the bipolar plasma cluster ions generated on the surface of the ceramic plate. This paper is important as it reveals some clues as to what happens when corona products interact with allergen molecules.

Due to the ability of corona products to destroy the structurally different group 1 and group 2 Dermatophagoides allergens and also Fel $\mathrm{d} 1$, it has previously been suggested that the active corona products must follow a broad-spectrum method of destroying proteins, probably affecting the primary structure. In agreement with this are the results of Kawamoto et al.'s biochemical analysis of the treated JCP allergen samples, which shows that the total protein content was degraded when visualized with SDS-PAGE. This complete protein degradation explains the significant reductions observed in both rabbit-reactive IgG epitope binding and also human IgE epitope binding. Furthermore, we now have some data on the allergenicity of treated samples in vivo: subjects suffering from pollinosis were found to react less to ion-treated samples in both intracutaneous and conjunctival reaction tests.

In future research, identification of the corona product(s) responsible for the destruction of allergens would be the first logical step to take in order to increase the allergen-destroying efficacy of an ionizer. Although Kawamoto et al. state that only two species of ions were generated in their setup (hydrated $\mathrm{H}_{3} \mathrm{O}^{+}$and $\mathrm{O}_{2}{ }^{-}$), it is known that many highly reactive species are created in this process and each have a different half-life [4-6]. Experiments performed using atmospheres of different pure gases, which would create different corona products, in combination with biochemical techniques to determine the structural and chemical alterations of proteins could aid identification. Once the active species are identified it would then be possible to enhance an ionizer's production of these species. By increasing the production of active corona products, whilst reducing harmful ozone production (e.g., by heating the corona electrode and modifying the electrode configuration of the ionizer [7]), the allergen content of the domestic environment could be reduced more efficiently and safely. A trade-off between the maximum amounts of these desired products in the plasma discharge and the unwanted ozone could then be made.

Preliminary investigations have already been performed into whether this technique of allergen reduction would translate well into a practical application for the removal of allergens from the domestic environment. The results of chamber tests and in situ, room-scale tests have

\section{KARGER}

Fax +41613061234 E-Mail karger@karger.ch www.karger.com
(C) 2006 S. Karger AG, Basel

$1018-2438 / 06 / 1414-0311 \$ 23.50 / 0$

Accessible online at:

www.karger.com/iaa
Correspondence to: Dr. Neil Goodman

Fluent Science

Rue Emile Heylens, 10

BE-1082 Brussels (Belgium)

E-Mailgoodman@fluentscience.com 
shown that it is possible to attain large reductions in allergen concentrations using both experimental and commercially available ionizers [8]. However, much more research is necessary to determine the benefits of allergen destruction using ionizers under natural conditions in the domestic environment - particularly any clinical benefits.

This raises the question, however, of how ionizers should be most effectively and safely used to reduce indoor concentrations of allergens below the threshold needed to sensitize and elicit symptoms. One possibility is to incorporate ion-generating devices into vacuum cleaners and other household appliances and further modification of indoor ionizers could lead to a protocol of use that would be easier to follow safely. It would be unwise to have continuous use of present-day ionizers to reduce the allergen load in the house due to the fact that exposure to ozone is harmful - especially to individuals with allergies [9]. Even if the ionizers produce less ozone than that seen to induce airway inflammation in humans, long-term exposure to this gas would still be dangerous.

One example of minimizing ozone (and ion) exposure would be to only operate the ionizers when no one is present in the house; perhaps turning the product on automatically when nobody is home and turning it off again before anyone returned would be safer. This method would also reduce exposure to the ions themselves, which, although no abnormalities or clinical signs were seen in 20 rats when exposed to ions for $6 \mathrm{~h}$ a day for 28 days [3], are far from being conclusively proven to be biologically inert.

The development of professional, industrial, intensive treatments using corona discharge and carried out by specialists, similar to steam cleaning or treatment with liquid nitrogen, could be envisaged. In this way, carpets, soft furnishings and mattresses could be cleaned of allergens and house dust mites without exposure of patients or pets. Systems could be designed where, similar to liquid nitro- gen freezing, upholstered furniture is encased and subjected to high-power corona discharge. These systems would have to include a mechanism to ensure the penetration of the active corona products through the surface of the material to where the major dust reservoir and mites are found. As preliminary experiments have shown that corona products penetrate only poorly through fabrics to where the main domestic allergen reservoirs are found (in some cases by only $10 \mathrm{~mm}$ ), reducing the allergen load by this method would need to incorporate either artificially increasing the velocity of the ion wind (by the use of fans or by increasing the ambient pressure outside) or reducing the pressure within the encasement to allow the corona products to penetrate more deeply [2]. This is similar to the high pressure required for effective steam cleaning.

Properly controlled and organized clinical trials using placebo and active ionizers for long periods of time in the homes of allergy patients also need to be performed to determine any clinical benefits. Symptoms and medication requirements could be recorded in the patient's diary during the test period and also skin prick tests and other clinical markers for allergy could be recorded before, during and after the exposure period. In order for the results to be meaningful, however, outside variables such as season, the amount of exercise taken, other methods of cleaning the house, and other possible avenues of contact with allergen should be taken into account. It would also be interesting to compare present treatments used to reduce allergens in the domestic environment with this novel method using corona discharge.

In conclusion, corona discharge has been shown to destroy allergens from a number of sources. This technique could be used to reduce the allergenic load in the domestic environment and, for this to occur, future studies will see greater involvement and collaborations between the fields of immunology and electrostatics.

\section{References}

1 Goodman N, Hughes JF: The effect of corona discharge on Der p 1. Clin Exp Allergy 2002; 32:515-519.

2 Goodman N, Hughes JF: The effect of corona discharge on dust mite and cat allergens. J Electrostatics 2004;60:69-91.

3 Kawamoto S, Oshita M, Fukuoka N, Shigeta S, Aki T, Hayashi T, Nishikawa K, Ono K: Decrease in the allergenicity of Japanese cedar pollen allergen by treatment with positive and negative cluster ions. Int Arch Allergy Immunol 2006;141:313-321.
4 Taylor DM, Secker PE: Industrial Electrostatics: Fundamentals and Measurements. Taunton, Research Studies Press, 1994.

5 Bastien F, Haug R, Lecuiller M: Simulation sur ordinateur de l'évolution temporelle des ions négatifs de l'air. Application au cas de la décharge couronne négative. J Chim Phys 1975;72:105-111.

6 Pethig R: The physical characteristics and control of air ions for biological studies. J Bioelectricity 1983;2:15-35.
7 Lui L, Guo J, Li J, Sheng L: The effect of wire heating and configuration on ozone emission in a negative ion generator. J Electrostatics 2001;48:81-91.

8 Goodman N, Hughes JF: Long-range destruction of Der $\mathrm{p} 1$ using experimental and commercially available ionizers. Clin Exp Allergy 2002;32:1613-1619.

9 Peden DB, Woodrow S, Devlin RB: Ozone exposure has both a priming effect on allergen-induced responses and an intrinsic inflammatory action in the nasal airways of perennially allergic asthmatics. Am J Respir Crit Care Med 1995;151:336-345. 